kehaviour of argon above the critical point is important. There is no single fluid phase but two distinct states of matter even though there is no visible discontinuity.

Commenting on the models of Dr. Egelstaff and Dr. Wilson, Prof. Bernal suggested that the presence of globules could be detected by the use of small-angle scatter and an estimate of their size obtained from viscosity measurements.
Turning next to alloys, Prof. Bernal put forward the idea that they could be considered as emulsions and cited as evidence the presence of the $\gamma$-phase in alloys of copper, silver or gold with zinc. In this phase the atoms are distributed at random over the lattice sites. However, the electron compound found in the gold-tin alloy system could be the cause of the structure observed in the X-ray diffraction patterns.

B. R. ORTON

\title{
RADIOACTIVE CHEMICALS, LABELLED COMPOUNDS AND RADIATION SOURCES
}

$\mathrm{F}^{\mathrm{a}}$ OR most purposes, particularly in engineering and radiotherapy, where the radiological properties of radioisotopes are of paramount importance, it is generally sufficient to refer to the tabulated physical data given in Part I of The Radiochemical Manual for a suitable choice of radioactive material. However, in the case of radioactive tracers for use in the biological sciences, chemical technology and related fields, the purity, chemical stability and the characteristic behaviour of the isotopes as radioactive chemicals are ofton the decisive factors, and for these Part 2 of the Manual*, which has recently been published and which deals with the chemical aspects of radioactive materials, must be consulted.

The range of radioactive chemicals is very extensive, and Part 2 has therefore been limited to a number of articles on selected topies likely to be useful to users of radioactive tracers. The examples referred to are drawn largely from the practical experience of the scientific staff at the Radiochemical Centre at Amersham. The contents of Part 2 consist of three sections, dealing with the sources of radioactive chemicals, some characteristic fuatures of the chemicals, and notes on labelled compounds of the elements carbon, hydrogen, sulphur, phosphorus, iodine and chlorine.

The first section contains four chapters in which the distinctive features which affect the manufacture of radioactive reagents are outlined. Radiological protection and remote handling frequently limit the types of chemical manipulation which can be carried out. The half-life of the isotope is the important factor in the organization of supply and distribution, and the chemical scale of manufacture which rarely exceeds one gram is a unique feature. The distinction between primary isotopes and labelled compounds is clearly drawn and the various methods of preparation are explained. Isotopic labelling and its wider implications, the synthesis of labelled compounds by chomical and biological methods and some of the unresolved problems such as the isotopic labelling of natural products by biosynthesis, are also discussed.

Chapters 5-7, which form the second section, are concerned with the concepts of purity and its determination, instability, and the behaviour of minute quantities and dilute solutions of radioactive chemicals. The final six chapters in the third section refer individually to compounds of each of the six elements which are most commonly concerned in tracer experiments. Carbon-14, which cmits only $\beta$-particles, is produced by means of a reactor in adequate quantities and at high specific activity, and, since it has a half-life of 5,760 years, it is a most favourable tracer isotope. It has been used for the intensive study of biochemical processes and has now virtually displaced the stable isotope carbon-13 and the short-lived $(20 \mathrm{~min})$ radioactive isotope carbon-11. Tritium-labelled compounds are now second in importance to carbon-14 compounds in biochemical tracer work. but tritium is not used as a hydrogen tracer but as an accessory tracer for a carbon atom or a molecule or a molecular fragment. Tritium is available in quantity and high specific activity (up to $29 \mathrm{c} . / \mathrm{m}$.amp) and its half-life of 12.3 years is conveniently long.

Sulphur-35 is the only practicable label for sulphur atoms. Its half-life is $87 \cdot 2$ days, and although it can be produced by direct neutron irradiation, it is better extracted as sulphate at high specific activity from neutron-irradiated potassium chloride. The main groups of chemical in which sulphur-35-labelled compounds are of interest are listed in the Manual. Phosphorus.32 is relatively easy to prepare. Its half-life is $\mathbf{1 4 \cdot 2}$ days and its main applications are in inorganic fertilizers, nerve gases, systemic insecticides, and plasticizers. Iodine-131 is in abundant supply from irradiated tellurium or from fission products, but its short half-life prevents stockholding of iodine-labelled compounds. Other isotopes of iodine, iodine-132, iodine-125 and iodine-124, are also available. Chlorine-36 has a long half-life of $3.03 \times 10^{5}$ years and is a pure $\beta$-emitter of medium energy. Its production is expensive and its specific activity is low, less than $4 \mathrm{mc} . / \mathrm{m}$.amp. Chlorino, however, is an important industrial chemical and therefore chlorine-36 has val. uable applications. It is also in many ways an ideal radio-isotope for use in the teaching of practical chemistry.

Part 2 of the Manual concludes with thirteen tables of synthetic routes to labelled compounds, a glossary of terms used, and numerous references to published work relating to the topics discussed and the tables. For details of the products, radioactive chemicals and radioactive sources, and services provided by the Radiochemical Centre the reader is referred to the catalogue, Radioactive Products $\dagger$, available on request from the Centre. S. Weintroub

* The Radiochemical Manual. Part 2: Radioactive Chemicals. Pp. v+78. (Amershasn: The Radioehemical Centre, 1963.) 258.

+ Radioactive P'roducts: Radioisotopes, Labelled Compounds, and Radiation Sources. Pp. 119. (Amersham: The Radiochenical Centre, 1962.)

\section{RESEARCH INTO TECHNICAL EDUCATION}

T HE report of a working party, with Prof. A. V. Judges as chairman, set up at the second session of a eonference on June 6,1961 , presided over by Dr. Dunsheath, and attended by representatives of the University of London, the Regional Advisory Council for Tochnological Education (London and Homo Counties), local authorities, technical colleges and research institutions, has now been published under the title Research into
Technical Education*. The working party was charged, inter alia, with considering priorities in research in this field and, apart from the specific proposals for research which are detailed, the report gives in its introduction an admirable survey of the present position and of the

* Research into Technical Education: Report of a Working Party. Pp. 34. (London: The Regional Advisory Council for Techuological Education. Jondon and Hone Counties, 1963.) 58. 